\title{
PRESIDENTIAL ADDRESS, APRIL 6, 1951 THE DIAGNOSTIC SIGNIFICANCE OF PAIN
}

BY

\author{
SIR HENRY COHEN
}

In choosing my subject for this address I have been influenced not only by the many recent advances in our knowledge of the mechanisms of pain, which are not yet woven into the fabric of clinical medicine, but also by my experience in both teaching and practice which leads me to believe that there is a diminishing appreciation in these days of high-powered diagnostic instruments, of the help which a careful analysis of pain, the most common presenting symptom of disease, can give in diagnosis. And this is of special significance and importance in the " rheumatic diseases ", where words and names have too readily displaced understanding; if this be doubted I shall sustain my thesis by a brief historical note.

Hippocrates used the term "arthritis" for joint diseases, but the name " rheumatism" was introduced by Galen 1,800 years ago. It derived from the then prevalent humoral pathology and was intended to indicate that the disease arose from a flow of peccant humors (Latin, rheuma, a flux). Hence all diseases thought to be due to such a discharge were labelled " rheumatic", and this term was applied not only to diseases of joints but also to diseases of serous membranes, e.g. rheumatic pleurisy, and to surface discharges, e.g. rheumatic catarrh. It was these "drops" of fluid into the joints and tissues which gave us the name "gout" (Latin, gutta, a drop). It is to Sydenham that we owe the conception of diseases as specific entities, a reversion in essence to the ancient demoniacal conception of disease. For Sydenham, diseases were " to be reduced to certain and determinate kinds, with the same exactness as we see it done by botanic writers in their treatises of plants ", and they were held to possess " certain distinguishing signs which nature has particularly affixed to every species". As a natural historian of disease, Sydenham recognized the constantly recurring patterns in the clinical pictures of acute rheumatism, of chorea, and of gout. Gradually the causes of many forms of arthritis were unmasked and the term " rheumatism " began to be used only for those painful diseases of the locomotor system-of joints, muscles and tendons, bursae, fascia, and bony skeleton-for which no specific and necessary causes could be found. Although the pain, swelling, and limited mobility of joints in arthritis left little doubt about the site of disease, in the so-called " non-articular " rheumatic states, a host of names was introduced, such as fibrositis, fasciomyositis, panniculitis, neuritis, etc., of which it could in truth be said that usually they simply 
masked ignorance of the cause of the painful malady from which a patient was suffering. No anatomical change, no functional or metabolic disturbance, and no pathology was customarily observed, though many hypotheses were formulated. Hence it is that, if we are not to " surrender judgment to the fascination of a name ", we must as physicians take heed of, and not simply pay lip service to, Hilton's injunction that " every pain has its distinct and pregnant signification if we will but carefully search for it ".

The first point to be stressed is that pain is a percept; it is subjective and we are, therefore, largely dependent on the patient's description of his pain for its interpretation. He must be a good witness and we must patiently pursue our task of exploring its site and radiation, its quality, its intensity, and the various modifications and alterations of bodily activity or external environment which excite, aggravate, or alleviate it. Since it is a percept, both the strength of the stimulus giving rise to pain, and the sensitivity of the central receptor mechanism on which nerve impulses impinge, will determine its intensity. Stick a pin in one patient and he says stolidly, "It's a prick"; but another will scream and withdraw violently. So we cannot judge the intensity of pain by the patient's reaction to it. Indeed, all objective signs which accompany pain give equivocal responses, so that little fruitful work on this subject has emerged from laboratory animal experiments. Only in man is the investigation of pain likely to yield useful data, though their interpretation may well be difficult.

For pain, as for all sensations, we can, without entering into the philosophical implications of Cartesian dualism, recognize two stages in perception. The first is anatomical: a noxious stimulus to pain receptors in the various tissues or organs of the body gives rise to an impulse which traverses a pathway through the peripheral nerve or sympathetic chains to spinal root ganglia; from there it passes to a cell station in the posterior horns, and thence through the anterior commissure to the opposite antero-lateral (spino-thalamic) tract, along which it ascends through the brain stem to the thalamus; its cortical associations are not yet clearly defined. When it reaches its cell stations in the brain, electrical and biochemical changes take place, but these are not pain. In some way, which remains obscure, there occurs at this stage in the normal, sentient, conscious being, an awareness of pain in a particular location which has its special quality and intensity.

The anatomical chain plays an important part in determining where pain is felt and also its quality. If we apply a noxious stimulus to any part of a nerve trunk containing pain fibres, pain will be referred to the area of distribution of the peripheral receptors of that nerve; for example, whether we faradize the ulnar nerve at the elbow or in the axilla, pain will be localized to the little finger and adjacent half of the ring finger; and if a prolapsed intervertebral disk irritates the left 4th lumbar root, pain will be felt down the left leg in the area of the peripheral distribution of this root. Like all root pains this distribution is segmental.

But there are other pains of segmental distribution which for us are of greater import. Briefly these arise from deep structures-muscles and bones, deep joints, and viscera. All these deep tissues and organs receive their sensory nerve supply 
from certain segments of the spinal cord, e.g. the stomach from $T_{7}-T_{9}$, the small intestine from $T_{9}-T_{11}$, and so forth. Disease in these deep structures gives rise to pain vaguely localized in the same segments at varying depths from the surface, and commonly in only part of the segment; hence cardiac disease may give pain in the arm (from $T_{1}$ ), diaphragmatic disease in the shoulder (from $\mathrm{C}_{4}$ ), kidney disease in the lower abdomen and groin (from $T_{10}-L_{1}$ ). I have dealt with the probable mechanism of this segmental distribution of pain in my Lettsomian Lectures (Cohen, 1948a). Segmental pain of this type may arise in an intervertebral disk lesion from disease of the disk itself, and can easily be differentiated from that component of the pain in a prolapsed disk which is due to irritation of the emerging spinal nerve; intervertebral arthritis, inflammatory lesions, strains and tears of muscles and tendons give rise to similar segmental pain, not involving the whole segment if mild, but becoming more widespread in its segmental distribution in its more intense forms.

Thus pains of the same segmental distribution may arise from disease of any structure-muscle, joint, periosteum, bone, or viscus - which is innervated from this segment, and its localization alone will not decide which segmental structure is diseased. This, as we shall shortly discuss, is disclosed by ascertaining what factors excite, aggravate, or alleviate the pain.

Before proceeding with this, however, I would remind you that although patients use a variety of adjectives to describe the quality of their pain, such as burning, aching, stabbing, tearing, throbbing, lightning, stitch-like, etc., there are essentially two main qualities of pain though intermediate varieties may be found.

The first or superficial pain is pricking, or if prolonged, burning; it is accurately localized and causes but little systemic disturbance. It arises when relatively superficial nerve endings are stimulated in the skin or subcutaneous tissues, in the mucous membrane of the mouth or pharynx, in the synovia of superficial joints, in periosteum, and in serous membranes. It occurs also when the nerve trunk conveying fibres from superficial structures is directly invaded by disease. Muscular rigidity is an uncommon accompaniment except when joints or serous membranes are involved; then it occurs as a protective reflex.

The second type of pain is deep; whatever its intensity, from mild to excruciating, it is usually accompanied by nausea (and if severe by vomiting)-a " sickening pain ", by lowering of blood pressure, by sweating, and even by collapse; it is associated with rigidity of those muscles receiving their motor nerve supply from the segment of the cord bombarded by noxious influences from the diseased structure. Deep pain is, as we have earlier observed, difficult to localize accurately; it has a diffuse segmental distribution. It arises from muscles and tendons, deep periosteum and joints, and from viscera, and it may arise also when the nerve trunk carrying " pain" fibres from deep structures is affected by disease. Deep pain has the qualities here described, whether it arises from musculo-skeletal tissues or from viscera. By its character alone its source cannot be determined.

It would be wrong to regard these two qualities of pain as clear-cut types. They merge into one another; the nearer the surface the diseased structure, the more 
closely the pain experienced corresponds to the superficial type; the deeper from the surface, the more is the quality of pain of the deep type.

We can then recognize four main origins of pain:

(i) from the skin and superficial tissues-e.g. buccal and pharyngeal mucous membrane, tibial periosteum, serous membranes, and superficial joints. This is pricking and burning in quality; the site of the noxious stimulus is well localized; there are no significant systemic reactions to the pain; muscular rigidity is rare, but may occur as a protective reflex.

(ii) from nerves or spinal roots. When these are mixed and receive fibres from both superficial and deep tissues, both superficial and deep pain may be felt in the area of distribution of the superficial nerve fibres, or segmentally if deep fibres be involved; when a spinal root is affected, the superficial and deep pain are felt segmentally; there is often an associated hypalgesia or analgesia and other signs of interruption of conduction in the nerve trunk, e.g. muscle wasting and abolition of reflexes.

(iii) from deep structures, including viscera, muscles, tendons, deep joints, periosteum, and bone. The pain is of deep, aching quality with segmental distribution, reflex muscle spasm, and systemic reactions.

(iv) from cord or brain, when the paths conveying pain impulses within the cord or brain are involved in disease, e.g. in new growths of the cord or thalamus. Here pain is often constant though subject to exacerbations without obvious cause, and when the thalamus is affected there may be marked emotional concomitants; the pain is often burning in type and may be associated with dysaesthesia and hypaesthesia; it extends over many segments, and sometimes almost half the body is involved on the contra-lateral side.

Clearly, unless a careful analysis is made of the pain so that the offending structure can be accurately indicted, much pain will pass as "rheumatic". Hence the importance of discovering exactly from where the pain is arising.

Though the criteria just described will help us to determine whether the pain arises from superficial or deep disease, and in what segment or segments of the body the offending structures lie, they do not help us to specify the exact organ or tissue involved. To achieve this we must pass to another observation, namely, What are the factors which excite, aggravate or alleviate the pain? For example, pain in the epigastrium may occur both from myocardial disease resulting in anoxia, or from peptic ulcer; the quality, localization and intensity of pain may be identical in both conditions, but the fact that the former is brought on by exertion and relieved by rest and trinitrin, whereas the latter is related to meals and eased by alkalies, firmly differentiates them. Similarly, pain in the groin may be due to a small hernia, to kidney or ovarian disease, to arthritis of the hip, or a host of other causes, but if we find that movement of the hip joint is limited and that it brings on the pain we are justified in looking to the hip as its origin. In the same way we can recognize pain arising from muscles, ligaments, and tendons, not only by local tenderness, but also by the effects of stretching or muscle contraction. I have elsewhere discussed how we may differentiate the various causes of backache by carrying out such tests (Cohen, 1948b). There is, however, one further observation of clinical importance which has often escaped notice, and yet is significant in the rheumatic field. 
Some years ago, I pointed out that, just as anaesthetization of the superficial area in which pain from deep-seated disease is localized can abolish or diminish the pain, so irritation, by injection of hypertonic saline or blistering, of the superficial area in the segmental distribution of a diseased viscus can give rise to pain in that area though it had never earlier been a seat of pain (Cohen, 1947). Let me give an example. A patient suffering from angina of effort experiences pain in the lower sternal region and inner side of his left arm on walking 150 yards up a steady, slight incline; hypertonic saline injected into the right infra-clavicular region or blistering of the right elbow caused pain to appear in these areas after walking 80-100 yards up the same incline, and before his usual anginal pains had appeared. If, therefore, the patient has more than one lesion in the same or contiguous segments, e.g. angina and spondylitis, the pain of either may be more easily induced and thus treatment of the spondylitis can lessen the anginal tendency and vice versa.

I expressed doubts earlier about the value of objective tests designed to assess the intensity of pain; these tests have included various forms of algesiometer, and the injection of hypertonic saline solution, or the burning of a uniform area of skin with radiant heat, to determine the area and duration of discomfort. Yet it is clinically of signal importance to be able to decide about the intensity of pain and the patient's reaction to it. Certain rough tests can be applied. Does the pain interfere with work or sleep ? Will aspirin control it or are stronger analgesics needed? The main factor to be appraised in assessing the significance of the intensity of pain is the patient's personality, for here we are faced with an everrecurring problem in diagnosis, which is sometimes roughly posed by the phrase"Is the pain "real"?" Now this question has no meaning unless the word " real" be defined. To the victim all pain is "real" in the sense that he suffers; except, of course, to the malingerer who deliberately and consciously feigns illness in the hope of reward. But since pain is a percept, it may arise because the central receptor mechanism (" psyche") is so sensitive that a stimulus, sufficiently slight to be ignored by a normal person, is experienced as "an unbearable pain ", or pain may even be a major complaint with no overt noxious stimulus. In so far as we identify the word " real" with an adequate organic cause (i.e. the presence of a diseased area capable of giving rise to adequate noxious stimuli) there are pains which are not " real ", in that many patients complain in bitter terms of severe pains for which we can find little or no adequate physical basis, and which we tend to attribute to the excessive receptivity of the sensorium; these are best named "psychogenic" pains.

We must not overlook, of course, the primary neuralgias which occur in the absence of physical signs of disease, but their character and distribution help us easily to differentiate them from "psychogenic" pains. The differentiating features of the primary neuralgias are these:

(i) The pains are paroxysmal, stabbing, and shock-like, in bouts lasting a few seconds separated by intervals of complete freedom from pain though of profound fear of its return, because of its excruciating severity;

(ii) stimulation of certain defined " trigger points" will excite a paroxysm of pain; 
(iii) there is no evidence of disease of the nerve nor signs of interference with its motor, sensory, or secretory functions;

(iv) the onset is usually in middle or old age;

(v) attacks recur at intervals over many years without significant spread;

(vi) the distribution of pain corresponds exactly to the anatomical course of the affected nerve.

How then can we recognize pain which is predominantly psychogenic ? The diagnosis must be based on positive features and not simply on the absence of organic disease, though this may well raise the suspicion in the first place. Among the criteria which I have found helpful are the following:

(i) The pain is almost invariably disabling, in the sense that it interferes with or prevents work, duty, or even pleasure.

(ii) It does not conform to any anatomical distribution. Indeed, its variable sites from day to day, or from hour to hour, are strikingly manifest, and it is associated with many contradictory phenomena, e.g. the painful area which is at one moment exquisitely sensitive to touch may be pressed upon without demur if the patient's attention is directed elsewhere.

(iii) It may be excited by fatigue or exhaustion, either mental or physical, but especially mental; pain due to organic disease may lead to exhaustion but does not follow it.

(iv) It commonly results from emotional stresses, conflicts, frustration, and neglect.

(v) It is commonly associated with other psychogenic disturbances, e.g. claustrophobia, and a constitutional inadequacy or predisposition to mental instability.

(vi) There is a history of manifold consultations, of innumerable investigations (the papers on which the results of these tests are recorded are often the presenting sign !), and of operations which have failed to benefit the patient. Such terms as " dropped stomach", " adhesions ", " inflammation of the ovary", etc., are names to which the patient has become so wedded that it is difficult to elicit the symptoms without the constant reiteration of a pet diagnostic label (but it should be remembered that there must be a first doctor whom the patient consults and that then this test will clearly not apply; he will be both lucky and wise if he avoids being the fount of iatrogenic illness !).

(vii) The patient almost always complains of the treatment meted out by former doctors, their negligence, and their ignorance (beware lest you be the next victim of his ire !).

None of these criteria is diagnostic but together they often paint an unmistakable picture.

Under what circumstances do these primarily psychogenic pains appear? Mainly in hysteria and the anxiety states but occasionally as a manifestation of a frank psychosis.

Using the word hysteria in its restricted sense of a psychogenic reaction designed to achieve a specific and personal aim, especially that of enabling the patient to escape from a difficulty, dilemma, or danger, or to fulfil a desire, of whose motivation he is in varying degrees unaware, we can recognize three types of pain in this category. Firstly, where there is a complaint of disease, and an hysterical version of the pain and other symptoms and signs which might be thought to be associated with it-so-called simple conversion hysteria; this is rare and easily recognized. Secondly, where old organic disease, e.g. injury, has been symptomless for years, but symptoms re-appear when the patient is faced with stress or hazard. Thirdly, where an injury, normally trivial, gives unduly prolonged disability and pain 
in circumstances where stress and strain may be met with on recovery or where compensation for injury is hopefully, if subconsciously, anticipated.

The pains of anxiety states may well be due, in part, to muscle contraction and spasm-the somatic expression of the increased nervous tension which is occasioned by fear. Too often a depressive psychosis is overlooked as a cause of persistent psychogenic pain, though few would fail to appreciate the origin of the bizarre pains described with unrestrained verbal embellishments by the paranoidal valetudinarian and hypochondriac.

I have sought to show that the problems presented by the clinical analysis of pain can be solved only by those who look at the "whole " man. Those whose major field of medical practice is rheumatology must beware of the compartmental outlook, for their diagnostic field is general medicine. They must adopt the "synoptic" view and decide, if pain be the presenting symptom, whether it is dominantly physical or psychogenic. They must be prepared to spend time on taking the history and making a full examination, so that they can determine what accessory procedures, provided by $x$ rays and clinical pathology, are necessary to reach a complete diagnosis, but they must not be bemused by the minor deviations from the normal which may be revealed by diagnostic machines, but which cannot possibly be related to the patient's complaints.

I do not claim to have presented any novel thesis; nor, indeed, were I able to do so, would this occasion have been appropriate. I have sought to review the more recent advances in our knowledge of pain and the help they give to its clinical interpretation, so that yet another step may be taken towards fulfilling Hilton's belief that " every pain has its distinct and pregnant signification if we will carefully search for it".

Cohen, H. (1947). Lancet, 2, 933.

\section{REFERENCES}

(1948a). Trans. med. Soc. Lond., 60, 1.

(1948b). "British Surgical Practice,", vol. 2, p. 1. Butterworth, London.

\section{HEBERDEN ROUND}

The Heberden Round was conducted by Professor L. S. P. Davidson in the Edinburgh Royal Infirmary on May 10, 1951. Eight cases were shown in the East Medical Theatre. The first four cases illustrated that pigmentation of the skin due to excess production of melanin can occur in four entirely unrelated diseases.

Case 1, Mrs. E.B., aged 32, pregnant.-She presented the typical clinical features of Addison's disease including pigmentation of the skin in the accepted situations. This was her second pregnancy, and the disease appeared to have followed immediately after the first pregnancy. She was treated with DOCA and salt. She was then put on to cortisone and at the time of the meeting was taking $15 \mathrm{mg}$. daily with DOCA twice 